\title{
PERCEPCIÓN DE LA ACEPTACIÓN Y EL RECHAZO DE LOS IGUALES EN ESCOLARES DE EDUCACIÓN PRIMARIA
}

\author{
Carolina Cedeño Coppiano \\ Universidad de Málaga \\ Carmen Barajas Esteban \\ Universidad de Málaga \\ barajas@uma.es \\ María José Linero Zamorano \\ Universidad de Málaga
}

\section{RESUMEN}

Recepción Artículo: 17 mayo 2021

Admisión Evaluación: 17 mayo 2021

Informe Evaluador 1: 26 mayo 2021

Informe Evaluador 2: 28 mayo 2021

Aprobación Publicación: 02 junio 2021

La aceptación que los niños/as reciben por parte de sus iguales puede ser muy desigual, así como la percepción que tienen de la aceptación recibida. Tanto la aceptación real como la precisión (o los sesgos) con que la perciben pueden tener consecuencias en la normal adaptación de los niños/as a su entorno social. El objetivo de este estudio es explorar la relación entre la aceptación/rechazo que reciben de sus iguales niños y niñas de Educación Primaria y la percepción de tal aceptación/rechazo, comparando los perfiles de las distintas categorías sociométricas. El estudio se llevó a cabo con una muestra de 97 niños/as (50 niñas y 47 niños) de 6 a 12 años, pertenecientes a centros educativos de Educación Básica de Quito (Ecuador). Se administró un cuestionario sociométrico, a partir del cual se estimó para cada participante su estatus sociométrico (popular, rechazado, controvertido, ignorado, promedio) y medidas de percepción de la aceptación y del rechazo, tanto generalizada como diádica. Los resultados indican que los niños/as rechazados sobrevaloran la aceptación global recibida (piensan que tienen más aceptación de la que realmente tienen); y que, en cuanto a la percepción diádica (0 percepción exacta de compañeros/as concretos que los eligen o rechazan), los niños/as ignorados subestiman la aceptación de los escasos compañeros que los eligen, mientras que los populares y promedio subestiman los casos de rechazo que reciben. Los resultados aportan claves interesantes para el diseño de situaciones educativas en las que los escolares tengan la oportunidad de comprobar y ajustar la percepción de la aceptación y/o rechazo de sus compañeros/as.

Palabras clave: aceptación/rechazo entre iguales; percepción sociométrica; categorías sociométricas; educación primaria 


\title{
PERCEPCIÓN DE LA ACEPTACIÓN Y EL RECHAZO DE LOS IGUALES EN ESCOLARES DE EDUCACIÓN PRIMARIA
}

\begin{abstract}
Perception of peer acceptance and rejection in elementary school children. The acceptance that children receive from their peers can be very uneven, as well as the perception they have of the acceptance received. Both the actual acceptance and the accuracy (or biases) with which they perceive it can have consequences on children normal adaptation to their social environment. The objective of this study is to explore the relationship between the acceptance/rejection that primary school children receive from their peers and the perception of such acceptance/rejection, comparing the profiles of the different sociometric categories. The study was carried out with a sample of 97 children (50 girls, 47 boys) aged between 6 and 12 years, recruited from educational centers of Basic Education in Quito (Ecuador). A sociometric questionnaire was administered, from which their sociometric status (popular, rejected, controversial, ignored, average) and measures of perception of acceptance and rejection, both generalized and dyadic, were estimated for each participant. The results show that rejected children overestimate the global acceptance received (they think they have more acceptance than they really have); and that, regarding the dyadic perception (or exact perception of specific peers who choose or reject them), ignored children underestimate the acceptance of the few peers who choose them, while the popular and average children underestimate the cases of rejection they receive. These results provide interesting clues for the design of educational situations in which schoolchildren have the opportunity to check and adjust the perception of peer acceptance and /or rejection.
\end{abstract}

Keywords: peer acceptance; peer rejection; sociometric perception; sociometric categories;

primary education

\section{INTRODUCCIÓN}

Este estudio está enfocado en analizar cómo perciben la aceptación y el rechazo que reciben de sus compañeros/as los niños y niñas de 6 a 12 años en función de su propio estatus sociométrico en el grupo de iguales.

Las relaciones sociales de los niños/as con sus iguales en el ámbito escolar constituyen un contexto fundamental para su desarrollo social y emocional. De ahí que en el marco de la psicología del desarrollo y de la educación haya una larga tradición de estudio de la aceptación y el rechazo que los niños/as reciben de sus compañeros/as, y de sus repercusiones en la adaptación social y académica. Son algo menos numerosos los estudios sobre cómo perciben los escolares la aceptación y el rechazo que reciben de sus iguales, aun cuando se trata de una habilidad de comprensión social muy valiosa para las interacciones sociales cotidianas (BlanchHartigan, Andrzajewski y Hill, 2012).

La técnica más habitualmente empleada para evaluar la aceptación en el grupo de iguales, o la preferencia entre los iguales como compañero de juego o amigo, es la de nominación de iguales (Bellmore y Cillesen, 2003; De Bruyn, Cillessen y Wissink, 2010; Morrow, M. T., Hubbard, J. A., Sallee, M. L., Barhight, L. R., Lines, M. M., y Rubin, R. M., 2015; Sureda, García-Bacete y Monjas, 2009). Mediante esta técnica pueden establecerse cinco tipos de estatus sociométrico (Coie, Dodge y Coppotelli, 1982), según la combinación de nominaciones positivas y negativas recibidas: popular, controvertido, promedio, rechazado e ignorado. El perfil propio de los niños/as populares es el de una buena adaptación escolar, un predominio de comportamiento prosocial y de autoevaluación positiva (Hutteman, Nestler, Wagner, Egloff, y Back, 2015; Mohanan y Booth-LaForce, 2016; Van Hoorn, Van Dijk, Meuwese, Rieffe y Crone, 2016); en el otro extremo, Ios niños/as rechazados muestran dificultades para adaptarse a las demandas escolares, una actividad social limitada y/0 no prosocial, y autoevaluación más negativa (Bierman, 2004; García-Bacete, Sureda, y Monjas, 2010; Martín y Muñoz de Bustillo, 2009; Mohanan y Booth-LaForce, 2016). La relación entre una mayor aceptación social y un mejor ajuste en el contexto escolar podría deberse, al menos en parte, a que el estatus sociométrico influye en la percepción de aceptación, lo que a su vez contribuye al ajuste social (Malloy, Albright y Scarpati, 2007).

Los primeros estudios sobre percepción sociométrica datan de la década de los 50; si bien, es desde la década de los 90 cuando se ha mantenido una cierta continuidad en el estudio de este aspecto de la cognición social. 
En estos estudios, se ha establecido una distinción principal entre precisión perceptiva y sesgo perceptivo. La precisión perceptiva es la medida en que uno puede predecir el grado de aceptación y/o rechazo que recibe por parte de un grupo determinado; cuando se evalúa mediante técnicas de nominaciones de iguales, la precisión perceptiva sería el grado de acuerdo entre la puntuación real recibida y la que se espera recibir (Portillo y Barajas, 2016). El sesgo perceptivo se refiere a la dirección y a la magnitud de la imprecisión perceptiva (Smith, Van Gessel, David-Ferdon y Kistner, 2013). Así, hay sesgo positivo de aceptación cuando se sobreestima la aceptación que uno tiene en el grupo (la puntuación total de aceptación esperada es mayor que la recibida); y hay sesgo negativo cuando se subestima (la puntuación total esperada es menor de la que se recibe). En el mismo sentido se definen la precisión y el sesgo perceptivo de rechazo.

En conjunto, las investigaciones sobre percepción sociométrica revelan que ciertos patrones de sesgos en las percepciones globales que los niños tienen de sus relaciones están asociados a dificultades de adaptación mientras que otros están asociados a funcionamiento adaptativo. En el primer caso, se ha encontrado, por ejemplo, que el sesgo positivo en la percepción de la aceptación está asociado con la conducta agresiva (David y Kistner, 2000; Lynch, R.J., Kistner, J.A., Stephens, H.F. y David Ferdon, C., 2016; Morrow et al., 2015; Neal, Neal y Cappella, 2014; Stephens, H.F., Kistner, J.A. y Lynch, R.J., 2015; White y Kistner, 2011), y con el TDAH (Hoza, B., Gerdes, A.C., Hinshaw, S., Arnold, L.E., Pelham, W.E., Molina, B. y Wigal, T., 2004); y que el sesgo negativo en la percepción de aceptación está asociado a síntomas depresivos (Campbell y Fehr, 1990; Morrow et al., 2015; Stephens et al., 2015) y a la soledad (Putarek, V. y Keresteš, G., 2016). En el segundo caso, se ha encontrado, por ejemplo, que el sesgo positivo de percepción de aceptación está asociado con el éxito académico (Gallardo y Barrasa, 2016; Rubin, K.H., Bukowski, W.M. y Laursen, B., 2009; Wentzel, K.R., Donlan, A. y Morrison, D., 2012) y con la estabilidad en la amistad (Badalay, Schwartz y Gorman, 2012). Dadas estas asociaciones, considerar las discrepancias entre la aceptación social y la percepción de aceptación parece valioso para identificar a niños y adolescentes tanto en situación de riesgo de consecuencias socio-emocionales negativas (Gallardo et al., 2016) como en condiciones especialmente favorables para su adaptación social y académica.

En este sentido, es muy pertinente al evaluar la auto-percepción social distinguir entre precisión y sesgo perceptivo generalizado versus diádico.

La precisión perceptiva generalizada refleja la impresión global de un niño/a de la aceptación que recibe en un grupo (cuánto gusta en general a sus compañeros/as), mientras que la precisión diádica refleja las predicciones específicas acerca de a qué compañeros/as en particular gusta (Malloy y Cillessen, 2008). Así, el sesgo en la percepción generalizada captura el grado en que un niño sobreestima o infraestima lo que gusta en global a sus iguales, mientras que el sesgo diádico se dirige a la sobreestimación o infraestimación de la aceptación recibida por compañeros/as concretos (Boor-Klip, Cillessen y Van Hell, 2014; Morrow et al., 2015). Ambas medidas de precisión y sesgo tienen mucho sentido, pero las medidas diádicas ofrecen estimaciones más precisas de consenso (Bellmore y Cillessen, 2003; Cillessen y Bellmore, 1999). Un niño/a puede percibir con precisión el grado global en que es aceptado o rechazado por sus compañeros, pero fallar en identificar a qué niños/as concretos gusta, mostrando una alta precisión generalizada pero una baja precisión diádica. Lo mismo puede ocurrir con el sesgo: podría subestimar el rechazo a nivel grupal y al mismo tiempo sobrestimarlo de niños concretos. Tanto las medidas de percepción generalizada como diádica otorgan estimaciones de consenso, pero cabe recalcar que la diádica concede mayor precisión en cuanto a las nominaciones determinadas (Bellmore y Cillessen, 2003; Boor-Klip, et al. 2014; Cillessen y Bellmore, 1999).

En cuanto a la percepción diádica, González y García-Bacete (2010), establecen una distinción interesante entre las medidas de Ajuste Perceptivo y Realismo Perceptivo. El Ajuste Perceptivo es una medida de la precisión con que percibe la aceptación o el rechazo que realmente se recibe de compañeros/as concretos; las nominaciones (positivas o negativas) no percibidas constituyen un sesgo negativo, o subestimación, en la percepción diádica. Por su parte, el Realismo Perceptivo es una medida de medida de lo ajustado de las expectativas de ser aceptado por compañeros/as concretos; las nominaciones (positivas o negativas) esperadas 


\section{PERCEPCIÓN DE LA ACEPTACIÓN Y EL RECHAZO DE LOS IGUALES EN ESCOLARES DE EDUCACIÓN PRIMARIA}

y en realidad no recibidas, constituyen falsas percepciones (de aceptación o de rechazo), o un sesgo de sobreestimación, en la percepción diádica.

En conjunto, las medidas de percepción generalizada y de percepción diádica de Ajuste y de Realismo perceptivo, pueden revelar información única y muy precisa del perfil de percepción de la aceptación y del rechazo de los escolares.

El objetivo general de este estudio es explorar la relación entre la aceptación/rechazo que reciben de sus iguales niños y niñas de Educación Primaria y la percepción de tal aceptación/rechazo. Este objetivo general se concreta en analizar el perfil perceptivo de la aceptación y del rechazo que presenta cada grupo sociométrico, precisando entre percepción generalizada y percepción diádica, y, dentro de esta entre Ajuste-subestimación y Realismo-sobreestimación.

\section{MÉTODO}

\section{Participantes}

El estudio se llevó a cabo con una muestra de 97 escolares (50 niñas y 47 niños) de Educación Básica, escolarizados en centros educativos de Quito (Ecuador), correspondientes a cuatro clases (de segundo, cuarto, quinto y sexto grados, respectivamente). El rango de edad de los participantes es de 6 a 11 años ( $M=9$ ). En términos de estatus sociométrico, la muestra incluye $10.30 \%$ populares $(n=10), 35.05 \%$ promedios $(n=34)$, $23.71 \%$ controvertidos ( $n=23), 15.46 \%$ rechazados $(n=15), 15.46 \%$ de ignorados $(n=15)$.

\section{Instrumentos}

Se administró un cuestionario sociométrico siguiendo el método de nominación de pares, con algunas variaciones respecto a la versión original de Coie, et al. 1982. Sobre el listado de todos los compañeros/as de clase, a cada participante se le pide que indique, sin establecer un límite de nominaciones:

a) "Los compañero/as con los que más te gusta jugar"

b) "Los compañeros/as con los que menos te gusta jugar"

c) "Los compañeros/as a los que más les gusta jugar contigo"

d) "Los compañeros/as a los que menos les gusta jugar contigo"

A partir de las nominaciones que emiten todos/as los participantes, se obtienen tanto los indicadores de aceptación y rechazo social entre iguales como los indicadores de percepción de la aceptación y del rechazo.

\section{Medidas de aceptación y rechazo}

A partir de las nominaciones de aceptación y de rechazo (preguntas a y b) se obtienen los índices sociométricos de: Nominaciones positivas recibidas (NPR), Nominaciones negativas recibidas (NNR), Impacto social (IS; suma de las nominaciones positivas y negativas recibidas), Preferencia social (PS; diferencia entre las nominaciones positivas y las negativas recibidas). Los indicadores de Preferencia social e Impacto social se combinaron para obtener el estatus sociométrico de cada participante de (según directrices de SOCIOMET: González y GarcíaBacete, 2010):

- Popular: Preferencia social positiva y alto impacto social.

- Controvertido: Preferencia social neutra y alto impacto social.

- Rechazado: Preferencia social neutra y alto impacto social.

-Ignorado: Baja preferencia social y bajo impacto social.

- Promedio: Preferencia social media e impacto social medio.

\section{Medidas de percepción de la aceptación y del rechazo}

A partir de las preguntas c) y d) se obtuvieron para cada participante los índices de percepción de la aceptación y del rechazo. Se estimó tanto la percepción generalizada como la percepción diádica. 


\section{Percepción generalizada.}

El Índice de percepción generalizada de aceptación se calculó dividiendo la cantidad de nominaciones positivas esperadas (multiplicada por cien) entre la cantidad real de nominaciones positivas recibidas. Una puntuación de 100 indica una precisión absoluta en la percepción generalizada de aceptación (100\%); las puntuaciones superiores a 100 indican el grado de sobrevaloración de la aceptación real; las puntuaciones inferiores a 100 indican el grado de infravaloración de la aceptación real.

De modo similar, el Índice de percepción generalizada de rechazo se calculó dividiendo la cantidad de nominaciones negativas esperadas (multiplicada por cien) entre la cantidad real de nominaciones negativas recibidas. Una puntuación de 100 indica una precisión absoluta en la percepción generalizada de rechazo (100\%); las puntuaciones superiores a 100 indican el grado de sobrevaloración del rechazo real; las puntuaciones inferiores a 100 indican el grado de infravaloración del rechazo real.

\section{Percepción diádica.}

Para la percepción diádica, se obtuvieron dos indicadores diferentes (siguiendo el procedimiento de SOCIOMET: González y García-Bacete, 2010).

\subsection{Indice de Ajuste Perceptivo.}

El índice de ajuste perceptivo de aceptación es una medida de la precisión con que percibe la aceptación que realmente recibe de compañeros/as concretos. Se calculó dividiendo las nominaciones positivas acertadas (multiplicado por 100) entre las nominaciones positivas recibidas (es decir, de todas las nominaciones positivas que recibe, cuántas realmente esperaba). Por ejemplo, un índice de ajuste perceptivo de aceptación de 60 indica que de la aceptación (nominaciones positivas) realmente recibida, percibe el $60 \%$. El valor complementario (hasta el 100\%) es una medida de la subestimación de la aceptación diádica (en el caso del ejemplo anterior, el $40 \%$ de la aceptación real no es percibida, por lo que se subestima).

De modo similar, el índice de ajuste perceptivo de rechazo es una medida de la precisión con que percibe el rechazo que realmente recibe de compañeros/as concretos. Se calculó dividiendo las nominaciones negativas acertadas (multiplicado por 100) entre las nominaciones negativas recibidas (es decir, de todas las nominaciones negativas que recibe, cuántas realmente esperaba). Por ejemplo, un índice de ajuste perceptivo de rechazo de 60 indica que del rechazo (nominaciones negativas) realmente recibido, percibe el $60 \%$. El valor complementario (hasta el 100\%) es una medida de la subestimación del rechazo diádico (en el caso del ejemplo anterior, el 40\% del rechazo real no es percibido, por lo que se subestima).

\subsection{Indice de Realismo Perceptivo.}

El índice de realismo perceptivo de aceptación es una medida del realismo de las expectativas de ser aceptado por compañeros/as concretos. Se calculó dividiendo las nominaciones positivas acertadas (multiplicado por 100) entre las nominaciones positivas esperadas (es decir, de todas las nominaciones positivas que espera, cuántas realmente recibe). Por ejemplo, un índice de realismo perceptivo de aceptación de 60 indica que de la aceptación esperada realmente recibe el 60\%. El valor complementario (hasta el 100\%) es una medida de la sobreestimación de la aceptación diádica (en el caso del ejemplo anterior, el 40\% de la aceptación esperada no la recibe realmente, por lo que la sobreestima).

De modo similar, el índice de realismo perceptivo de rechazo es una medida del realismo de las expectativas de ser rechazado por compañeros/as concretos. Se calculó dividiendo las nominaciones negativas acertadas (multiplicado por 100) entre las nominaciones negativas esperadas (es decir, de todas las nominaciones negativas que espera, cuántas realmente recibe). Por ejemplo, un índice de realismo perceptivo de rechazo de 60 indica que del rechazo esperado realmente recibe el 60\%. El valor complementario (hasta el 100\%) es una medida de la sobreestimación del rechazo diádico (en el caso del ejemplo anterior, el $40 \%$ del rechazo esperado no lo recibe realmente, por lo que lo sobreestima). 


\section{PERCEPCIÓN DE LA ACEPTACIÓN Y EL RECHAZO DE LOS IGUALES EN ESCOLARES DE EDUCACIÓN PRIMARIA}

\section{Procedimiento}

Los responsables académicos, directores y profesores de los escolares fueron informados de los objetivos y del procedimiento del estudio. El primer encuentro se generó con los padres y madres de familia para compartir los consentimientos y asentimientos informados; a partir de ahí se generó un encuentro virtual con cada estudiante, de aproximadamente 15 minutos, para recabar la información del cuestionario sociométrico.

Las entrevistas fueron acordadas en horarios accesibles para las familias y los estudiantes, lo cual permitió la administración eficiente de los tests. Esta investigación fue aprobada por el Comité de Ética de la Pontificia Universidad Católica del Ecuador, sede Quito.

Análisis de datos

Los datos fueron analizados con el paquete estadístico IBM SPSS Statistics 26. Se aplicaron análisis $t$ de student para muestras relacionadas para comprobar si había diferencias estadísticamente significativas entre las medias de percepción de aceptación y las de rechazo, para las medidas de: percepción generalizada, ajuste perceptivo-subestimación diádico y realismo perceptivo-sobreestimación diádico. Se aplicaron estos análisis para cada una de las categorías sociométricas: popular, controvertido, rechazado, promedio e ignorado.

\section{RESULTADOS}

Se muestran en primer lugar los resultados referidos a la percepción generalizada; en segundo lugar, los de las comparaciones de Ajuste Perceptivo - subestimación en la percepción diádica; y en tercer lugar los de Realismo perceptivo - sobreestimación, también en la percepción diádica.

Percepción generalizada de aceptación y de rechazo según estatus sociométrico

La Tabla 1 muestra las medias de los índices de percepción generalizada de aceptación y de rechazo para cada grupo sociométrico.

Los resultados de los análisis $t$ de student de comparación de medias muestran que los niños/as rechazados muestran valores significativamente muy superiores en la percepción generalizada de aceptación respecto a la percepción generalizada del rechazo $(t(14)=6,626, p=.000)$.

La única categoría sociométrica que muestra diferencias significativas es la de rechazados, para el resto de las categorías sociométricas no se muestran resultados significativos.

Tabla 1 Medias y Desviaciones Típicas de las medidas de Percepción generalizada de la aceptación y del rechazo según Estatus Sociométrico

\begin{tabular}{lcc}
\hline Estatus sociométrico & $\begin{array}{c}\text { Percepción Generalizada } \\
\text { de Aceptación } \\
M(D T)\end{array}$ & $\begin{array}{c}\text { Percepción Generalizada de } \\
\text { Rechazo } \\
M(D T)\end{array}$ \\
\hline Popular & $65,75(17.87)$ & $120,0(154,91)$ \\
Promedio & $138,53(64,16)$ & $132,65(116,49)$ \\
Controvertido & $77,36(26,01)$ & $77,32(26,01)$ \\
Rechazado & $225,56(102,08)$ & $62,11(39,84)$ \\
Ignorado & $100(146,38)$ & $80,00(122,18)$
\end{tabular}

Subestimación de la percepción diádica de aceptación y de rechazo según estatus sociométrico

La Tabla 2 muestra las medias de los índices de subestimación de aceptación y de rechazo para cada grupo sociométrico.

Los resultados de los análisis $t$ de student de comparación de medias muestran que los niños/as populares muestran valores significativamente muy superiores en la subestimación de rechazo respecto a la subestimación de la aceptación $(t(9)=-7,011 p=.000)$. 
Otra de las categorías que muestra resultados significativos en la de promedios, revelando valores significativamente superiores en la subestimación del rechazo respecto a la subestimación de la aceptación $(t(33)=$ $6,055 p=.000)$.

La categoría rechazados muestra una tendencia a la significación, evidenciando una tendencia a la significación en la subestimación del rechazo respecto a la subestimación de la aceptación $(t(14)=-2,130 p=.051)$.

Para el resto de las categorías sociométricas no se muestran resultados significativos.

Tabla 2. Medias y Desviaciones Típicas de las medidas de Subestimación de la aceptación y del rechazo según Estatus Sociométrico. (Percepción diádica).

\begin{tabular}{lcc}
\hline $\begin{array}{l}\text { Estatus } \\
\text { sociométrico }\end{array}$ & $\begin{array}{c}\text { Subestimación } \\
\text { de la Aceptación } \\
M(D T)\end{array}$ & $\begin{array}{c}\text { Subestimación } \\
\text { del Rechazo } \\
M(D T)\end{array}$ \\
\hline Popular & $63,20(16,59)$ & $100.00(.000)$ \\
Promedio & $65,62(28,97)$ & $97,94(9,13)$ \\
Controvertido & $70,61(18,50)$ & $81,91(22,34)$ \\
Rechazado & $63,33(44,18)$ & $90,40(10,01)$ \\
Ignorado & $100.00(.000)$ & $95,53(17,29)$ \\
\hline
\end{tabular}

Sobreestimación de la percepción diádica de aceptación y de rechazo según estatus sociométrico

La Tabla 3 muestra las medias de los índices de sobreestimación de aceptación y de rechazo para cada grupo sociométrico.

Los resultados de los análisis $t$ de student de comparación de medias muestran que los niños/as populares tienen valores significativamente muy superiores en la sobreestimación del rechazo respecto a la sobreestimación de la aceptación $(t(9)=-7,813 p=.000)$.

Los resultados de los análisis $t$ de student de comparación de medias muestran que los niños/as promedios tienen valores significativamente superiores en la sobreestimación del rechazo respecto a la sobreestimación de la aceptación $(t(33)=-5,687 p=.000)$.

Para el resto de las categorías sociométricas no se muestran resultados significativos.

Tabla 3. Medias y Desviaciones Típicas de las medidas de Sobreestimación de la aceptación y del rechazo según Estatus Sociométrico. (Percepción diádica).

\begin{tabular}{lcc}
\hline $\begin{array}{l}\text { Estatus } \\
\text { sociométrico }\end{array}$ & $\begin{array}{c}\text { Sobreestimación } \\
\text { de la Aceptación } \\
M(D T)\end{array}$ & $\begin{array}{c}\text { Sobreestimación } \\
\text { del Rechazo } \\
M(D T)\end{array}$ \\
\hline Popular & $42,50(23,27)$ & $100,00(, 000)$ \\
Promedio & $74,38(22,15)$ & $98,06(7,88)$ \\
Controvertido & $56,17(29,00)$ & $70,30(34,12)$ \\
Rechazado & $77,80(28,16)$ & $78,80(26,98)$ \\
Ignorado & $100,00(, 000)$ & $95,53(17,29)$ \\
\hline
\end{tabular}




\section{PERCEPCIÓN DE LA ACEPTACIÓN Y EL RECHAZO DE LOS IGUALES EN ESCOLARES DE EDUCACIÓN PRIMARIA}

\section{DISCUSIÓN}

Este estudio examina la percepción de la aceptación y el rechazo de los iguales en escolares de Educación Primaria. El objetivo es explorar la relación entre la aceptación/rechazo que reciben de sus iguales niños y niñas de Educación Primaria y la percepción de tal aceptación/rechazo, comparando los perfiles de las distintas categorías sociométricas.

Tomando en cuenta las categorías sociométricas, se compararon las medias de la percepción generalizada, es decir, cuánto gusta de manera global a sus compañeros/as. El único grupo que arrojó diferencias significativas fue el de rechazados, mostrando valores superiores en la percepción generalizada de aceptación respecto a la del rechazo. Este grupo de niños/as ha recibido un mayor número de nominaciones negativas, por lo cual es curioso que ellos sobreestimen la aceptación y subestiman el rechazo, alejándose de una precisión en cuanto a su estatus dentro del grupo.

Sobre la precisión de la percepción diádica (predicciones específicas acerca de a qué compañeros/as gusta o no gusta), los niños/as populares muestran valores superiores en la subestimación de rechazo respecto a la de la aceptación, esperando tener menos nominaciones negativas de las que realmente reciben y dejando de percibir algunas nominaciones negativas de sus pares. Es interesante cómo perciben ser menos rechazados de lo que realmente son en su grupo de iguales.

En cuanto a la subestimación de la aceptación en este grupo, se evidencia que es menor (en relación con la del rechazo), sin embargo, demuestra que también esperan menos nominaciones positivas en su grupo de iguales, evidenciando que son más aceptados de lo que logran percibir. Estos niños/as no logran captar quiénes los aceptan en su grupo de iguales, por eso subestiman la aceptación. Las falsas percepciones pueden estar relacionadas al número de nominaciones que reciben, ya que al ser numerosas les resulta complejo identificar su posición dentro de la dinámica de grupo.

Otro de los resultados significativos en cuanto a la precisión de la percepción diádica se da en el grupo de promedios, mostrando mayor subestimación del rechazo que de la aceptación. Esperan menos nominaciones negativas de las que realmente reciben, dejando de identificar quiénes si los nominaron. Su subestimación es menor en cuanto a la aceptación, pero también esperan menos nominaciones positivas de las que reciben. Este grupo percibe su aceptación y su rechazo en menor medida de lo que realmente es.

Si bien el resto de las categorías no presenta resultados significativos en cuanto a la precisión de la percepción diádica, el grupo de niños/as rechazados muestra una tendencia a la significación, evidenciando una mayor subestimación del rechazo. Este dato es interesante, ya que ellos han sido nominados frecuentemente en negativo y, sin embargo, no logran percibir a dichos niños/as que los han nominado.

En cuanto a la sobreestimación de la aceptación y del rechazo, los resultados arrojaron datos interesantes sobre los niños/as populares. Sobreestiman mucho más el rechazo que la aceptación. Esperan recibir más nominaciones negativas de las que realmente reciben, demostrando una falsa percepción en cuanto a quiénes creen que los nominarían. Sobreestiman la aceptación en menor medida, pero también esperaran ser nominados en positivo por algunos de sus pares y esto no sucede.

También los niños/as promedios tienen valores significativamente superiores en la sobreestimación del rechazo respecto a la sobreestimación de la aceptación, esperando más nominaciones negativas de las que realmente reciben.

Otro hallazgo interesante, aunque no arrojó ser significativo en la comparación de medias, es que los niños/as ignorados tienen una precisión exacta de la aceptación, logrando estimar cuán aceptados son realmente dentro de su grupo de iguales (percepción generalizada). Esta identificación podría estar asociada a que no reciben un gran número de nominaciones por parte sus pares, pero a pesar de esto, ellos logran tener esta precisión.

Los resultados también son interesantes en la medida que pueden ser comparados con datos de otros estudios que están en la misma línea. En el estudio de Boor-Klip et al. (2014) se menciona respecto a la autopercepción social, que los estudiantes que son más rechazados tienen una percepción de la aceptación más 
precisa, probablemente porque son menos nominaciones entre las que escoger. Este dato es significativo en la medida que el único grupo que arrojó datos reveladores en la percepción generalizada dentro de esta investigación justamente fue el de los niños/as rechazados, pero esta vez, sobreestimando en gran medida la aceptación y alejándose de dicha precisión.

En conclusión, se evidencia que la subestimación y la sobreestimación es significativa en las categorías sociométricas de populares y promedios (percepción diádica). Los niños/as rechazados logran percibir de manera general su aceptación y rechazo, pero no necesariamente detectan quiénes los han nominado (percepción generalizada). Mientras que el grupo de los ignorados no presenta resultados significativos dentro de esta investigación.

En futuras investigaciones sería interesante completar el análisis del perfil perceptivo de cada categoría sociométrica introduciendo el estudio de las reciprocidades en las nominaciones positivas y negativas, así como el de las inversiones perceptivas, tanto de aceptación como de rechazo (o veces que se espera aceptación y se recibe rechazo, y a la inversa). Estos análisis implicarían determinar la correspondencia de las nominaciones entre los pares (negativas o positivas), así como precisar si dichas nominaciones son contrarias.

\section{FINANCIACIÓN}

Este trabajo ha sido financiado por el Plan Propio de Investigación de la Universidad de Málaga. Programa para la promoción de proyectos de investigación en Ciencias Sociales, Código: 2019_1.

\section{REFERENCIAS BIBLIOGRÁFICAS}

Badaly, D., Schwartz, D. y Gorman, A.H. (2012). Social status, perceived social reputations, and perceived dyadic relationships in early adolescence. Social Development, 21 (3), 482-500.

Bellmore, A. D., y Cillessen, A. H. N. (2003). Children's meta-perceptions and meta accuracy of acceptance and rejection by same-sex and other-sex peers. Personal Relationships, 10, 217233. https://doi.org/10.1111/1475-6811.00047

Bierman, K. L. (2004). Peer rejection: Developmental processes and intervention strategies. Guilford Press.

Blanch-Hartigan, D. Andrzejewski, S.A. y Hill, K.M. (2012). The effectiveness of training to improve per-son perception accuracy: A meta-analysis. Basic and Applied Social Psychology, 34, 483-498.

Boor-Klip, H. J., Cillessen, A. H. N., y Van Hell, J. G. (2014). Social understanding of high ability children in middle and late childhood. Gifted Child Quarterly, 58, 259-271. https://doi.org/10.1177/0016986214547634

Campbell, J.D. y Fehr, B. (1990). Self-esteem and perceptions of conveyed impressions: Is negative affectivity associated with greater realism? Journal of Personality and Social Psychology, 58, 122-133.

Cillessen, A. H. N., y Bellmore, A. D. (1999). Accuracy of social selfperceptions and peer competence in middle childhood. Merrill-Palmer Quarterly, 45, 650-676.

Coie, J., Dodge, K. A., y Coppotelli, H. (1982). Dimensions and types of social status: A cross-age perspective. Development Psychology, 18, 557-570. https://doi.org/10.1037/0012-1649.18.4.557

Crick, N. R., y Dodge, K. A. (1994). A review and reformulation of social information-processing mechanisms in children's social adjustment. Psychological Bulletin, 115, 74-101. https://doi.org/10.1037/00332909.115.1.74

David, C.F. y Kistner, J.A. (2000). Do positive self-perceptions have a "dark side"? Examination of the link between perceptual bias and aggression. Journal of Abnormal Child Psychology, 28 (4), 327-337.

de Bruyn, E. H., Cillessen, A. H., y Wissink, I. B. (2010). Associations of peer acceptance and perceived popularity with bullying and victimization in early adolescence. The Journal of Early Adolescence, 30(4), 543-566.

Gallardo, L. y Barrasa, A. (2016). Self-other agreement measures of acceptance in predicting academic achievement: A longitudinal analysis. Current Psychology.

García Bacete, F. J., Sureda García, I., y Monjas Casares, I. (2010). El rechazo entre iguales en la educación primaria: Una panorámica general. Anales de psicología. 


\section{PERCEPCIÓN DE LA ACEPTACIÓN Y EL RECHAZO DE LOS IGUALES EN ESCOLARES DE EDUCACIÓN PRIMARIA}

Gónzalez, J. y García Bacete, F. J. (2010). Manual de Uso del Sociomet. Madrid: TEA Ediciones. ISBN: 978-847174-921-5

Hoza, B., Gerdes, A.C., Hinshaw, S., Arnold, L.E., Pelham, W.E., Molina, B. y Wigal, T. (2004). Self-perceptions of competence in children with ADHD and comparison children. Journal of Consulting and Clinical Psychology, 72 (3), 382-391.

Hutteman, R., Nestler, S., Wagner, J., Egloff, B., y Back, M. D. (2015). Wherever I may roam: Processes of selfesteem development from adolescence to emerging adulthood in the context of international student exchange. Journal of Personality and Social Psychology, 108(5), 767.

Lynch, R.J., Kistner, J.A., Stephens, H.F. y David Ferdon, C. (2016). Positively biased self perceptions of peer acceptance and subtypes of aggression in children. Aggressive Behavior, 42 (1), 82-96.

Malloy, T. E., \& Cillessen, A. H. N. (2008). Variance component analysis of generalized and dyadic peer perceptions in adolescence. In N. A. Card, T. D. Little, y J. P. Selig (Eds.), Modeling dyadic and interdependent data in developmental and behavioural research (pp. 213-244). Mahwah, NJ: Erlbaum

Malloy, T. E., Albright, L., y Scarpati, S. (2007). Awareness of peers' judgments of oneself: Accuracy and process of metaperception. International Journal of Behavioral Development, 31, 603-610. https://doi.org/10.1177/0165025407080590

Martín, E., y De Bustillo, M. C. M. (2009). Un análisis contextual de la preferencia y el rechazo entre iguales en la escuela. Psicothema, 21(3), 439-445.

Monahan, K. C., y Booth LaForce, C. (2016). Deflected pathways: Becoming aggressive, socially withdrawn, or prosocial with peers during the transition to adolescence. Journal of research on adolescence, 26(2), 270285.

Morrow, M. T., Hubbard, J. A., Sallee, M. L., Barhight, L. R., Lines, M. M., y Rubin, R. M. (2015). Dyadic accuracy and bias in preadolescents' perceived peer relations: Associations with aggression, depression and peer victimization. Journal of Social and Personal Relationships, 7, 892-916. https://doi.org/10.1177/0265407515605907

Neal, J. W., Neal, Z. P., y Cappella, E. (2014). I know who my friends are, but do you? Predictors of self reported and peer inferred relationships. Child development, 85(4), 1366-1372.

Portillo, M., y Barajas, C. (2016). Teoría de la mente, aceptación entre iguales y auto-percepción social. Apuntes de psicología, 34(1), 47-58.

Putarek, V. y Keresteš, G. (2016). Self-perceived popularity in early adolescence Accuracy, associations with loneliness, and gender differences. Journal of Social and Personal Relationships, 33 (2), 257-274.

Rubin, K.H., Bukowski, W.M. y Laursen, B. (2009). Hand-book of Peer Interactions, Relationships, and Groups. Nueva York: Guilford Press.

Smith, S. D., Van Gessel, C. A., David-Ferdon, C., y Kistner, J. A. (2013). Sex differences in children's discrepant perceptions of peer acceptance. Journal of Applied Developmental Psychology, 34(2), 101-107.

Stephens, H.F., Kistner, J.A. y Lynch, R.J. (2015). The calculation of discrepancy scores in the context of biased self-perceptions of acceptance. Journal of Psychopathology and Behavioral Assessment, 37,442-453.

Sureda, I., García-Bacete, J. F., y Monjas, I. (2009). Razones de niños y niñas de diez y once años para preferir 0 rechazar a sus iguales [Reasons why ten- and eleven-year-old children prefer or reject their peers]. Revista Latinoamericana de Psicología, 2, 305-321. http://doi.org/10.14349/rlp.v41i2.382

van Hoorn, J., van Dijk, E., Meuwese, R., Rieffe, C., y Crone, E. A. (2016). Peer influence on prosocial behavior in adolescence. Journal of Research on Adolescence, 26(1), 90-100.

Wentzel, K.R., Donlan, A. y Morrison, D. (2012). Peer relationships and motivation at school. En A.M. Ryan y G.W. Ladd (Eds.), Peer Relationships and Adjustment at School (pp. 79-108). Charlotte, NC: IAP

White, B.A. y Kistner, J.A. (2011). Biased self-perceptions, peer rejection, and aggression in children. Journal of Abnormal Child Psychology, 39, 645-656. 